\title{
Optical spin-symmetry breaking for high-efficiency directional helicity-multiplexed metaholograms
}

\author{
Muhammad Ashar Naveed ${ }^{1}$, Muhammad Afnan Ansari', Inki Kim², Trevon Badloe $\mathbb{D}^{2}$, Joohoon Kim², Dong Kyo Oh², \\ Kashif Riaz', Tauseef Tauqeer', Usman Younis', Murtaza Saleem³, Muhammad Sabieh Anwar ${ }^{3}$, Muhammad Zubair', \\ Muhammad Qasim Mehmood ${ }^{1}$ and Junsuk Rho (i) 2,45
}

\begin{abstract}
Helicity-multiplexed metasurfaces based on symmetric spin-orbit interactions (SOls) have practical limits because they cannot provide central-symmetric holographic imaging. Asymmetric SOls can effectively address such limitations, with several exciting applications in various fields ranging from asymmetric data inscription in communications to dual side displays in smart mobile devices. Low-loss dielectric materials provide an excellent platform for realizing such exotic phenomena efficiently. In this paper, we demonstrate an asymmetric SOl-dependent transmission-type metasurface in the visible domain using hydrogenated amorphous silicon (a-Si:H) nanoresonators. The proposed design approach is equipped with an additional degree of freedom in designing bi-directional helicity-multiplexed metasurfaces by breaking the conventional limit imposed by the symmetric SOI in half employment of metasurfaces for one circular handedness. Two on-axis, distinct wavefronts are produced with high transmission efficiencies, demonstrating the concept of asymmetric wavefront generation in two antiparallel directions. Additionally, the CMOS compatibility of a-Si:H makes it a cost-effective alternative to gallium nitride $(\mathrm{GaN})$ and titanium dioxide $\left(\mathrm{TiO}_{2}\right)$ for visible light. The costeffective fabrication and simplicity of the proposed design technique provide an excellent candidate for highefficiency, multifunctional, and chip-integrated demonstration of various phenomena.
\end{abstract}

\section{Introduction}

Metasurfaces provide an efficient and miniaturized platform for nanophotonics due to their ability to tailor the wavefronts of light at the subwavelength scale. They have been applied in display applications ${ }^{1,2}$, communications $^{3}$, beam engineering ${ }^{4,5}$, security ${ }^{6}$, and data storage $^{7}$. Conventionally, in holography, computergenerated holography $(\mathrm{CGH})$ algorithms are used to produce the required phase and amplitude profiles of a

Correspondence: Muhammad Zubair (muhammad.zubair@itu.edu.pk) or Muhammad Qasim Mehmood (qasim.mehmood@itu.edu.pk) or JunsukRho (jsrho@postech.ac.kr)

${ }^{1}$ NanoTech Lab, Department of Electrical Engineering, Information Technology University (ITU) of the Punjab, Ferozepur Road, Lahore 54600, Pakistan

${ }^{2}$ Department of Mechanical Engineering, Pohang University of Science and Technology (POSTECH), Pohang 37673, Republic of Korea

Full list of author information is available at the end of the article

These authors contributed equally: Muhammad Ashar Naveed, Muhammad

Afnan Ansari, Inki Kim hologram $^{8}$. In metasurface-based holography, plasmonic structures have been used in both reflection ${ }^{9,10}$ and transmission $^{11,12}$. Li et al. proposed a multicolor metallic metasurface that can produce different images for the three primary colors, i.e., red, green, and blue. Crosstalk between kaleidoscopic holographic images is reduced using different optimal angles of incidence ${ }^{13}$. An ultrabroadband achromatic phase distribution is achieved by a catenary aperture-based plasmonic metasurface ${ }^{14}$. However, plasmonic metasurfaces that contain small metallic nanoresonators severely suffer from ohmic losses. As the ohmic losses lead to absorbance, the maximum theoretical efficiency decreases, while the measured efficiency is even lower due to fabrication defects ${ }^{15,16}$. This opens the door to exploring new materials to achieve highly efficient metasurfaces.

High-refractive-index dielectric materials are an ideal candidate for this purpose because they display strong

\section{(c) The Author(s) 2021}

(c) (i) Open Access This article is licensed under a Creative Commons Attribution 4.0 International License, which permits use, sharing, adaptation, distribution and reproduction cc) in any medium or format, as long as you give appropriate credit to the original author(s) and the source, provide a link to the Creative Commons license, and indicate if changes were made. The images or other third party material in this article are included in the article's Creative Commons license, unless indicated otherwise in a credit line to the material. If material is not included in the article's Creative Commons license and your intended use is not permitted by statutory regulation or exceeds the permitted use, you will need to obtain permission directly from the copyright holder. To view a copy of this license, visit http://creativecommons.org/licenses/by/4.0/. 
electric and magnetic resonances in the visible domain ${ }^{17-19}$. Silicon is a widely used material for highrefractive-index dielectrics. Yang et al. proposed a polysilicon-based metasurface to achieve maximum crosspolarization reflection and the required phase at operational wavelengths of $1367-1380 \mathrm{~nm}^{20}$. Zhou et al. proposed a crystalline silicon-(c-Si)-based metasurface with $47 \%$ efficiency and full $0-2 \pi$ phase control at the optimized working wavelength of $532 \mathrm{~nm}^{21}$. However, amorphous silicon (a-Si) exhibits very significant dielectric losses in the visible domain, which seriously reduces its efficiency ${ }^{22}$. Dielectric materials such as $\mathrm{GaN}$ and $\mathrm{TiO}_{2}$ are transparent in the visible domain with high-refractive indices. Ellipsometry data of $\mathrm{GaN}^{23}$ and $\mathrm{TiO}_{2}{ }^{24}$ strongly advocate their usage for fabricating efficient metalenses ${ }^{25}$ and metaholograms ${ }^{26}$. For example, a highly efficient and broadband metasurface using $\mathrm{GaN}$ nanopillars to produce wavefronts with arbitrary polarization based on given linearly polarized light has been presented ${ }^{27}$. Similarly, Khorasaninejad et al. demonstrated high-aspect-ratio $\mathrm{TiO}_{2}$ metalenses enabling diffraction-limited convergence at wavelengths of 660, 532, and $405 \mathrm{~nm}$ with transmission efficiencies of $66 \%, 73 \%$, and $86 \%$, respectively $^{28}$. However, an incompatibility with existing CMOS technology is the major drawback of these material platforms. Moreover, the designs based on these materials possess high-aspect ratios (up to 15 ) to achieve the required phase coverage, which leads to complex and costly fabrication processes ${ }^{25,29}$. To overcome these problems, a-Si:H-based metasurfaces have been proposed $^{30,31}$. The addition of hydrogen to noncrystalline silicon lowers the absorption coefficient while maintaining a high-refractive index, comparable to that of $\mathrm{GaN}$ and $\mathrm{TiO}_{2}$. Therefore, the proposed a-Si:H can deliver a CMOS-compatible platform for fabricating highrefractive-index dielectric metasurfaces. Other exciting dielectric materials, such as indium tin oxide (ITO) and silicon nitride $(\mathrm{SiN})$, have also been reported as potential candidates for implementation of high-efficiency metasurfaces operating in the visible domain ${ }^{32,33}$. However, the refractive indices of ITO $(\sim 2)^{32}$ and $\operatorname{SiN}(\sim 2)^{34}$ are lower than that of a-Si:H ( 3.25); therefore, nanorods made of ITO and $\mathrm{SiN}$ must be taller than those made of a-Si:H to achieve the required full phase modulation. Although ITO and $\mathrm{SiN}$ are well-known CMOS-compatible materials, a$\mathrm{Si}: \mathrm{H}$ promises simpler fabrication with smaller aspect ratios to achieve a similar functionality at the working wavelength.

Metasurfaces have shown great flexibility towards effective scaling and integration for multiple applications. However, most metaholograms only perform a single operation, i.e., they have half-space operation ${ }^{5,35}$. One way to realize bifunctional metasurfaces is to employ chiral structures that can utilize asymmetric transmission (AT) to design polarization-sensitive bioperational metadevices $^{36-38}$. Such chiral AT effects originate from the breaking of the mirror symmetry of the structures and have been observed in multilayer structures ${ }^{39,40}$. These structures are vulnerable to complex fabrication processes; any mismatch in subsequent layers drastically reduces their efficiency. Chen et al. proposed a bifunctional and bilayer metapolarizer that attained efficiencies of $17 \%$ and $15.5 \%$ in the backward and forward directions, respectively, at an operating wavelength of $735 \mathrm{~nm}^{41}$. Chen et al. presented 3D helical metallic nanoapertures to achieve a direction-multiplexed and polarization-sensitive metasurface that can generate two unique wavefronts in the forward and backward directions under circularly polarized incidence and linearly polarized incidence, respectively. The maximum measured transmission efficiency of this metasurface was $20 \%$ at a wavelength of $800 \mathrm{~nm}^{42}$. Similarly, Chen et al. employed cascaded anisotropic impedance metallic sheets with different geometries to achieve asymmetric transmission, enabling direction-sensitive functionalities with a peak efficiency of $80 \%$ at the operation frequency of $8.6 \mathrm{GHz}^{43}$. Zhang et al. demonstrated a single-layer spin-orbit-based metasurface exhibiting a very large AT in the mid-IR region ${ }^{44}$. However, the complexity of designing the proposed metasurfaces restricts scaling them down to the visible domain. Moreover, conventional metaholograms regulate the propagation of electromagnetic light by incorporating photon spin-orbit interactions based on the geometric phase. Such photon spin interaction-based metaholograms provide central-symmetric holography, which limits the helicity multiplexing approach. As a result, only $50 \%$ of the metadevice is active for a particular polarization handedness; therefore, the total efficiency of a geometric phase metadevice remains half regardless of its material properties.

Here, we propose an all-dielectric single-layer metasurface that enables asymmetric wavefront generation (AWG) for backward and forward incident circularly polarized light using a-Si:H nanoresonators. The phasevarying anisotropic antennas are spatially arranged for AWG such that the absence of mirror symmetry is maintained. The Pancharatnam-Berry (PB) phase originates from the rotation of the nanoresonators, and its value is reversed for the same circularly polarized light incident from different directions, while the retardation phase is direction-insensitive and depends on the physical dimensions and material properties of the nanoresonators $^{44}$. In our proposed all-dielectric metasurface, both the PB and retardation phases are merged such that two independent and arbitrary pieces of information can be multiplexed into one metasurface, engaging the entire metasurface (unlike traditional geometric or PB phase metasurfaces). This proposed metahologram yields two 


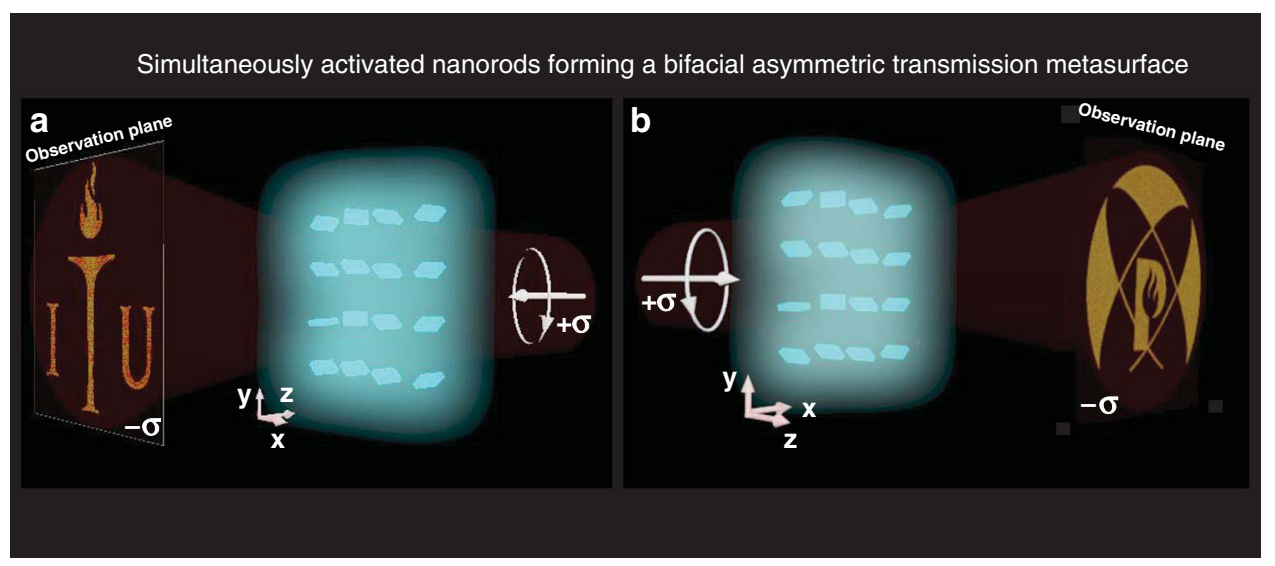

Fig. 1 Operation of the proposed asymmetric wavefront generation metasurface. Right circularly polarized light incident from the a backward direction and $\mathbf{b}$ forward direction on a hydrogenated amorphous silicon (a-Si:H) metasurface. A single metasurface can produce two holographic images (the logo of POSTECH or Information Technology University "ITU") depending on the propagation direction (see supplementary video part c).

holographic images, one from each side (backward and forward), depending on the light illumination direction, as depicted in Fig. 1. To prove this concept, a metasurface producing two highly efficient holographic images (logos of ITU and POSTECH) for antiparallel propagating circularly polarized $(\mathrm{CP})$ incident light is simulated and fabricated. This proposed multiplexing technique based on asymmetric spin-orbit interactions has great potential in fabricating bifunctional metadevices.

\section{Methods and results \\ Design methodology}

High-refractive-index nanoresonators have a strong localized effect on shaping the phase and wavefront of incoming light. We can utilize an array of such nanoresonators to fabricate a metasurface, where each nanoresonator independently acts as a half-wave plate (HWP) or nanowaveguide ${ }^{30,31,45}$. a-Si:H nanoresonators are used on top of a silicon dioxide $\left(\mathrm{SiO}_{2}\right)$ substrate, as illustrated in Fig. 2b. At the desired wavelength of $632.8 \mathrm{~nm}$, the mobility gap and extinction coefficient of conventional a$\mathrm{Si}$ are 1.7 and $0.47 \mathrm{eV}$, respectively. These values restrict its usage in the visible domain, as absorption becomes very prominent for wavelengths below $708 \mathrm{~nm}$. With the inclusion of hydrogen, the mobility gap and extinction coefficient of a-Si can be increased and decreased, respectively, as the hydrogen eliminates the gap states and passivates the unstructured bonds ${ }^{46}$. The ellipsometry data depicted in Fig. 2a show that the measured refractive index of the proposed a-Si:H is $3.25+0.047 \mathrm{j}$ at $632.8 \mathrm{~nm}$, which illustrates a significant decrease in loss compared to a-Si. The physical parameters (i.e., height $(\mathrm{H})$, length $(\mathrm{L})$, width (W), and periodicity (P)) of each unit cell are optimized to achieve the maximum cross-polarization transmission efficiency at the operational wavelength of
$632.8 \mathrm{~nm}$. The optimal periodicity while considering the Nyquist sampling criteria $(\mathrm{P}<\lambda / 2 \mathrm{NA}$, where $\lambda$ and NA indicate the operating wavelength and numerical aperture) is achieved at $290 \mathrm{~nm}$, as shown in Fig. 2c. The $\mathrm{L}$ and $\mathrm{W}$ of each nanoresonator are optimized using a parametric sweep while keeping P fixed at $290 \mathrm{~nm}$. The crosspolarization transmission ( $\mathrm{T}_{\text {cross }}$ ) efficiency for different $\mathrm{L}$ and $\mathrm{W}$ is shown in Fig. 2d. Four points are selected from the maximum $\mathrm{T}_{\text {cross }}$ and minimum $\mathrm{T}_{\text {co }}$ region (indicated by white dots) such that each nanoresonator has an incremental phase retardation of $\pi / 4$ compared to its neighbor. The basic building block of our proposed metasurface is an array of such high-refractive-index nanoresonators. The $\mathrm{H}$ and $\mathrm{P}$ of each nanoresonator are kept the same, while the $\mathrm{L}$ and $\mathrm{W}$ are changed to produce the required phase profile. The optimized $L$ and $\mathrm{W}$ for the four nanoresonators are as follows: $1(\mathrm{~W}=$ $60 \mathrm{~nm}, \mathrm{~L}=210 \mathrm{~nm}), 2(\mathrm{~W}=80 \mathrm{~nm}, \mathrm{~L}=200 \mathrm{~nm}), 3(\mathrm{~W}=$ $95 \mathrm{~nm}, \mathrm{~L}=210 \mathrm{~nm})$, and $4(\mathrm{~W}=110 \mathrm{~nm}, \mathrm{~L}=230 \mathrm{~nm})$.

The broadband cross- and co-polarization transmission analyses of the optimized nanoresonators along with their average values are depicted in Fig. 3a, b. All of the nanoresonators have their minimum co-polarization transmission and maximum cross-polarization transmission $(\eta)$ resonances at the optimized wavelength of $632.8 \mathrm{~nm}$. This is evident from the average simulated value for cross-polarization transmission at a wavelength of $632.8 \mathrm{~nm}$ of $\approx 63 \%$, while the average co-polarization transmission is limited to $\approx 6.2 \%$. Higher crosspolarization efficiencies can also be acquired at wavelengths of 532 and $405 \mathrm{~nm}$ by utilizing a supercell scheme with unit elements individually optimized at three different wavelengths $(632.8,532$, and $405 \mathrm{~nm})$.

Resonance modes are studied for the individual nanoresonators. Both electric and magnetic resonance 
a
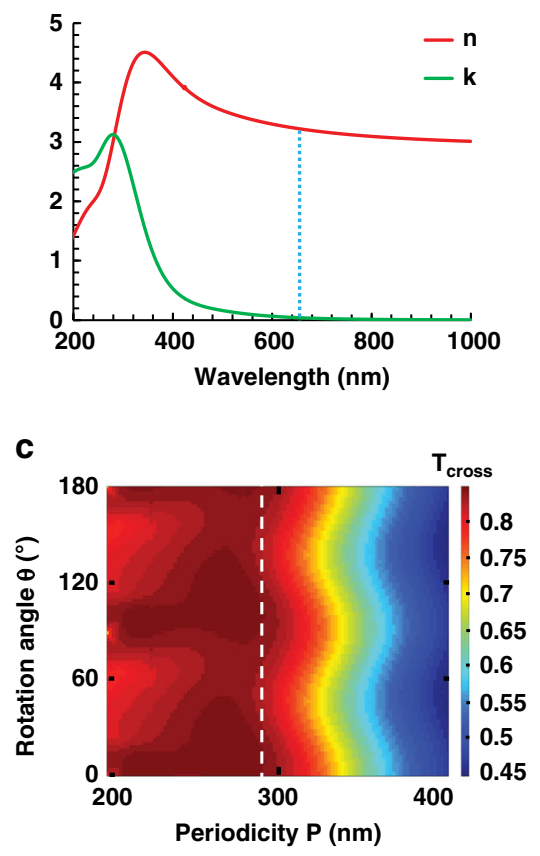

b
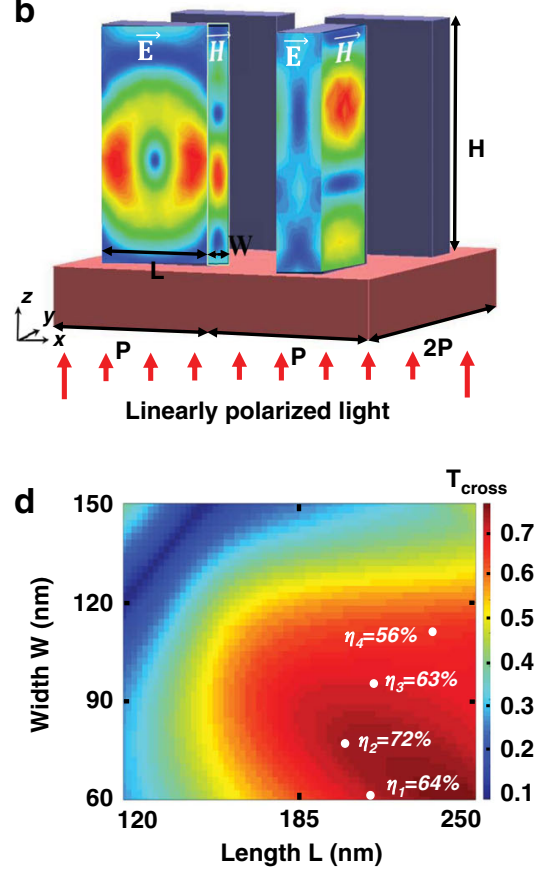

e

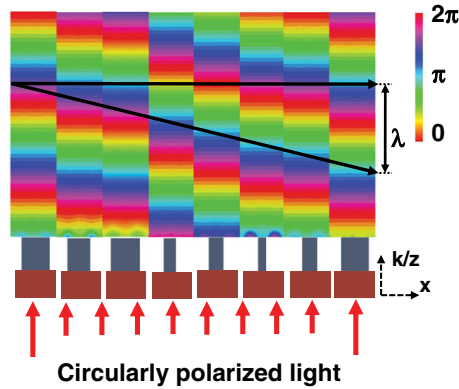

Fig. 2 Optimization of the a-Si:H nanorods. a Measured values of refractive index ( $\mathrm{n}$ ) and extinction coefficient ( $\mathrm{k}$ ) of a-Si:H; at $\lambda=632.8 \mathrm{~nm}$, the complex refractive index is $3.25+0.047$ j. $\mathbf{b}$ Unit-cell configuration composed of a-Si:H nanoresonators on a $\mathrm{SiO}_{2}$ substrate. The height $(\mathrm{H}=400 \mathrm{~nm})$ and period $(P=290 \mathrm{~nm})$ are constant, but the length $(L)$ and width $(W)$ are different for all nanoresonators, depending on the required phase. For the two front nanoresonators, the $\vec{E}$ and $\vec{H}$ fields under x-polarized light, which are well confined in the nanoresonators, are depicted. c Efficiency of cross-polarization transmission $\left(T_{\text {cross }}\right)$ for a range of $\mathrm{P}$ and rotation angles $(\theta)$ of the a-Si:H unit cell. The white dashed line at $\mathrm{P}=290 \mathrm{~nm}$ denotes where the average value of $T_{\text {cross }}$ is $~ 0.97$. $\mathbf{d}$ Efficiency of cross-polarization transmission ( $\left.T_{\text {cross }}\right)$ for different $L$ and $W$. Four points are selected such that they have the maximum transmission efficiency $\left(\eta\right.$ ) along with a mutual $45^{\circ}$ phase difference (white dots are marked at the respective positions). e Wavefront of all nanoresonators for cross-polarized transmitted light at a working wavelength of $632.8 \mathrm{~nm}$, obtained from full-wave simulations. $0-2 \pi$ phase coverage and complete control over the wavefront of the transmitted light are realized.

modes exist and are well confined within the nanoresonators. The a-Si:H nanoresonators show electric and magnetic resonance modes similar to those previously reported $^{47}$, but with greater intensity due to the lower extinction coefficient in the visible domain. The dielectric resonance modes of the optimized nanoresonators with respect to photon spin interactions are depicted in Supplementary Fig. S1.

The total phase gradient of a nanoresonator is a combination of the PB phase and retardation phase. The PB phase originates from the rotational alignment of the nanoresonators, while the retardation phase depends on the material properties and physical parameters of the nanoresonators. By merging these two independent phases, we can achieve an asymmetric $\mathrm{SOI}^{48}$. Each nanoresonator acts as an HWP and imparts a phase shift of $\phi \pm 2 \sigma \theta$ for the two orthogonal polarizations along its major and minor axes ${ }^{44,48}$. Here, $\theta$ defines the orientation angle of the nanoresonators with the $x$-axis, the sign \pm represents the forward and backward propagation direction of the incident light, and $\sigma$ represents the circular helicity of the incident light. Moreover, $\phi$ denotes the value of the retardation phase. We have carefully designed and optimized four unit cells with an incremental phase 

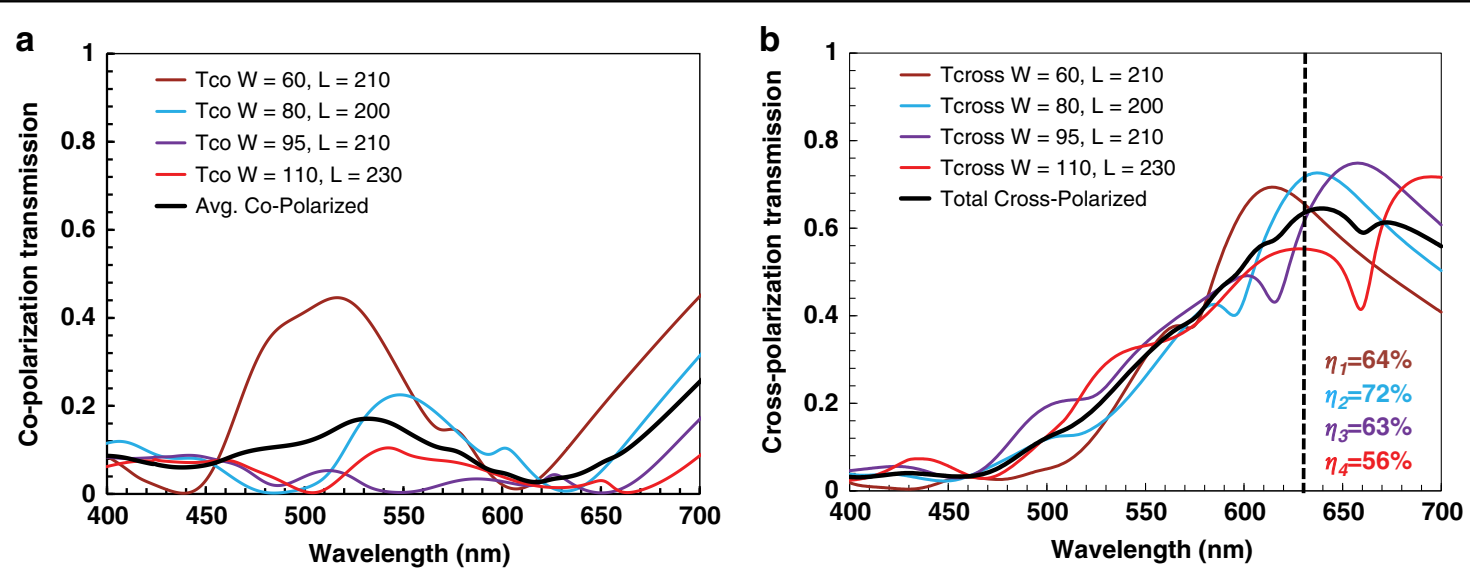

Fig. 3 Broadband analysis of co-polarization and cross-polarization efficiencies. a Co-polarization components of all four optimized unit cells along with their average co-polarization. At the optimized wavelength of $632.8 \mathrm{~nm}$, the average co-polarization transmission is minimized $(\approx 6.2 \%)$. b Cross-polarization components of the four optimized unit cells along with their average cross-polarization transmission ( $\eta$ ). At the operational wavelength, the average cross-polarization transmission is maximized $(\approx 63 \%)$. The black dashed line indicates the working wavelength of $632.8 \mathrm{~nm}$.

difference of $\pi / 4$ with respect to each other at the central operating wavelength of $632.8 \mathrm{~nm}$. For full phase coverage from $0-2 \pi$, these four unit cells are repeated with a $\pi / 2$ phase difference, as shown in Fig. 2 d.

The retardation phase and $\mathrm{PB}$ phase can be independently controlled because these nanoresonators have low crosstalk due to the interresonator spacing being less than half of the working wavelength and hence act as weakly coupled Fabry-Pérot resonators ${ }^{44,48}$. Each nanoresonator simultaneously contributes to the forward and backward phase profile of the metasurface. The selection of the nanoresonators and their orientation relative to the $x$-axis can be performed using the following equations (the proof is given in supplementary information: Section 2):

$$
\begin{aligned}
& \phi(x, y)=\frac{\psi_{b}(x, y)+\psi_{f}(x, y)}{2} \\
& \theta(x, y)=\frac{\psi_{b}(x, y)-\psi_{f}(x, y)}{4}
\end{aligned}
$$

Here, $\psi_{b}$ and $\psi_{f}$ represent the required phase profile distributions for backward- and forward-propagating circularly polarized light, respectively. Eight quantized levels of the calculated phase are defined for appropriate selection of the nanoresonators, with each being placed at the corresponding position on the metasurface with a specific rotation $\theta$.

\section{Experimental verification}

For realization of a metasurface-based hologram, the phase profile required for the output beam needs to be determined. The Gerchberg-Saxton (GS) algorithm is the simplest technique that can provide the amplitude of the incident field along with the desired phase pattern in the far-field ${ }^{49}$. Using the modified GS algorithm, phase matrices for two separate logo images (ITU and POST$\mathrm{ECH}$ ) were calculated separately. The phase matrix of one image (ITU) was selected for $\psi_{f}$, with that of the other image (POSTECH) being selected for $\psi_{b}$. These forward and backward phases were multiplexed according to Eqs. (1) and (2) for $\phi$ and $\theta$. The calculated values of $\phi$ were subdivided into eight quantized levels ranging from $0-2 \pi$ in ascending order. Each nanoresonator was employed at a corresponding position on the metasurface by comparing its phase with these quantized levels. The orientation of the nanoresonators was defined by the corresponding value of $\theta$.

To verify the proposed concept, initially, a modified GS scheme was used to calculate a $(1036 \times 1036)$ phase matrix for the two logos, which corresponds to an $\sim 300 \times$ $300 \mu \mathrm{m}^{2}$ asymmetric wavefront generation metasurface. The numerically calculated far-field images of these phase maps are depicted in Fig. 4a, b. Simulation results obtained by a finite-difference time-domain (FDTD)based full-wave solver are depicted in Fig. 4c, d. The ITU logo appears when RCP light impinges on the metahologram from the backward direction, and the POSTECH logo appears under RCP illumination from the forward direction.

Plasma-enhanced chemical vapor deposition (PECVD) was utilized to fabricate an $\approx 300 \times 300 \mu \mathrm{m}^{2}$ asymmetric wavefront generation metahologram. A 400-nm thick layer of a-Si:H was grown on top of a 500$\mu \mathrm{m}$ thick $\mathrm{SiO}_{2}$ substrate. The deposition rate of a-Si:H was maintained at $1.3 \mathrm{nms}^{-1}$ at $300{ }^{\circ} \mathrm{C}$ by controlling the flow rates of silane $\left(\mathrm{SiH}_{4}\right)$ and hydrogen $\left(\mathrm{H}_{2}\right)$ gases at 10 and $75 \mathrm{sccm}$, respectively. Then, electron-beam lithography was employed to define rectangular-shaped 

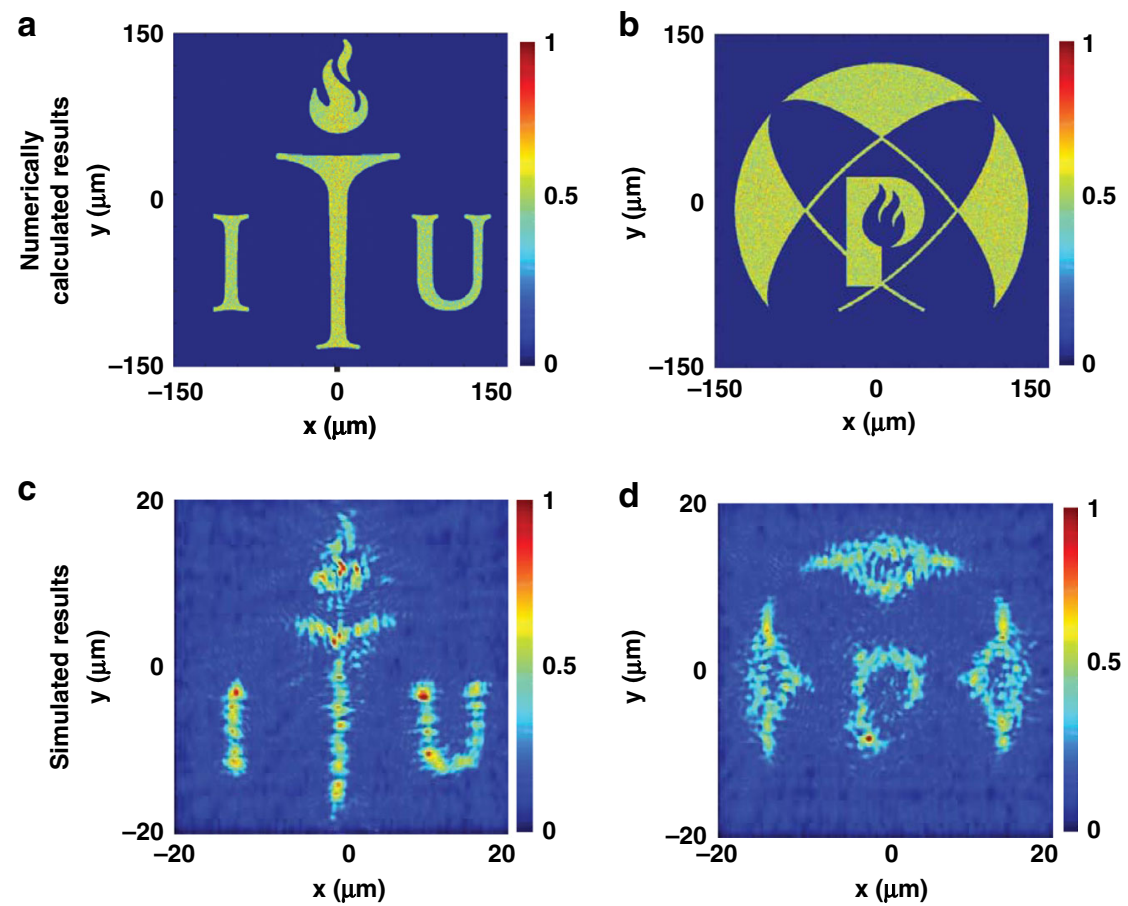

Fig. 4 Numerically calculated and simulated results for the asymmetric wavefront generation metasurface. Numerically calculated phase map for an $\approx 300 \times 300 \mu^{2}$ metahologram for RCP light incident in the $\mathbf{a}$ forward and $\mathbf{b}$ backward directions. Simulated results for a $40 \times 40 \mu m^{2}$ size Fourier-type metahologram obtained from finite-difference time-domain simulations (using FDTD Lumerical software) at a focal distance of $20 \mu \mathrm{m}$ for RCP light incident in the $\mathbf{c}$ backward and $\mathbf{d}$ forward directions.

a-Si:H nanoresonators on the patterned scanned positive photoresist. A 30-nm thick chromium (Cr) layer was deposited, followed by the lift-off process. The geometrical structures were transferred to the a-Si:H using the $\mathrm{Cr}$ mask and dry etching. A pictorial depiction of the fabrication steps for the asymmetric wavefront generation metaholograms along with final scanning electron microscope (SEM) images are presented in Fig. 5a, b.

The optical characterization results and corresponding setup used for the characterization of our fabricated AWG metahologram is depicted in Fig. 6a-d. A $5 \mathrm{~mW}$ $\mathrm{HeNe}$ laser was used as the incident light source at an operational wavelength of $632.8 \mathrm{~nm}$. A quarter-wave plate (QWP) along with a linear polarizer (LP) was used to covert the light into LCP or RCP light. This circularly polarized light was passed through the beam splitter (BS) and divided into two parts with a 50/50 ratio. The metasurface sample was positioned next to the beam splitter on a two-dimensional stage at the desired beam waist. One part of this light passed through the metasurface in the forward direction and produced one holographic image (the logo of POSTECH) as shown in Fig. 6a. When mirrors M1 and M2 were active, the rest of the light was reflected through them (represented by the blue path). Another beam splitter was employed to impinge the light onto the metasurface from the opposite side (antiparallel to the first case). A second hologram (the logo of ITU) appeared in the backward direction as presented in Fig. 6b. Two charge-coupled device (CCD) cameras were used to capture the images through a $\times 40$ objective lens (OL) and a tube lens (TL) on both sides as seen in Fig. 6c. In the complete characterization process, the sizes of both the incident and output optical beams were kept equal. The transmitted optical power was measured to obtain the transmission efficiency of the metahologram using a CCD camera. The incident power $\left(\mathrm{P}_{\text {in }}\right)$ from the source was measured directly with the CCD camera, while the cross-polarized optical power $\left(\mathrm{P}_{\text {cros-pol }}\right)$ was measured in the far-field of the metahologram. The total efficiency of the metahologram is calculated by the following formula [Eq. 3] (and measured efficiency at $632.8 \mathrm{~nm}$ wavelength is represented in Fig. 6d):

$$
\eta=\frac{\mathrm{P}_{\text {cros }-\mathrm{pol}}}{\mathrm{P}_{\text {in }}}
$$

The measured transmission efficiency for the designed asymmetric wavefront generation metahologram is $59 \%$. The reconstructed images of the POSTECH and ITU logos projected in the far-field by the metahologram are depicted in Fig. 6a, b. Both images are very clear and have 


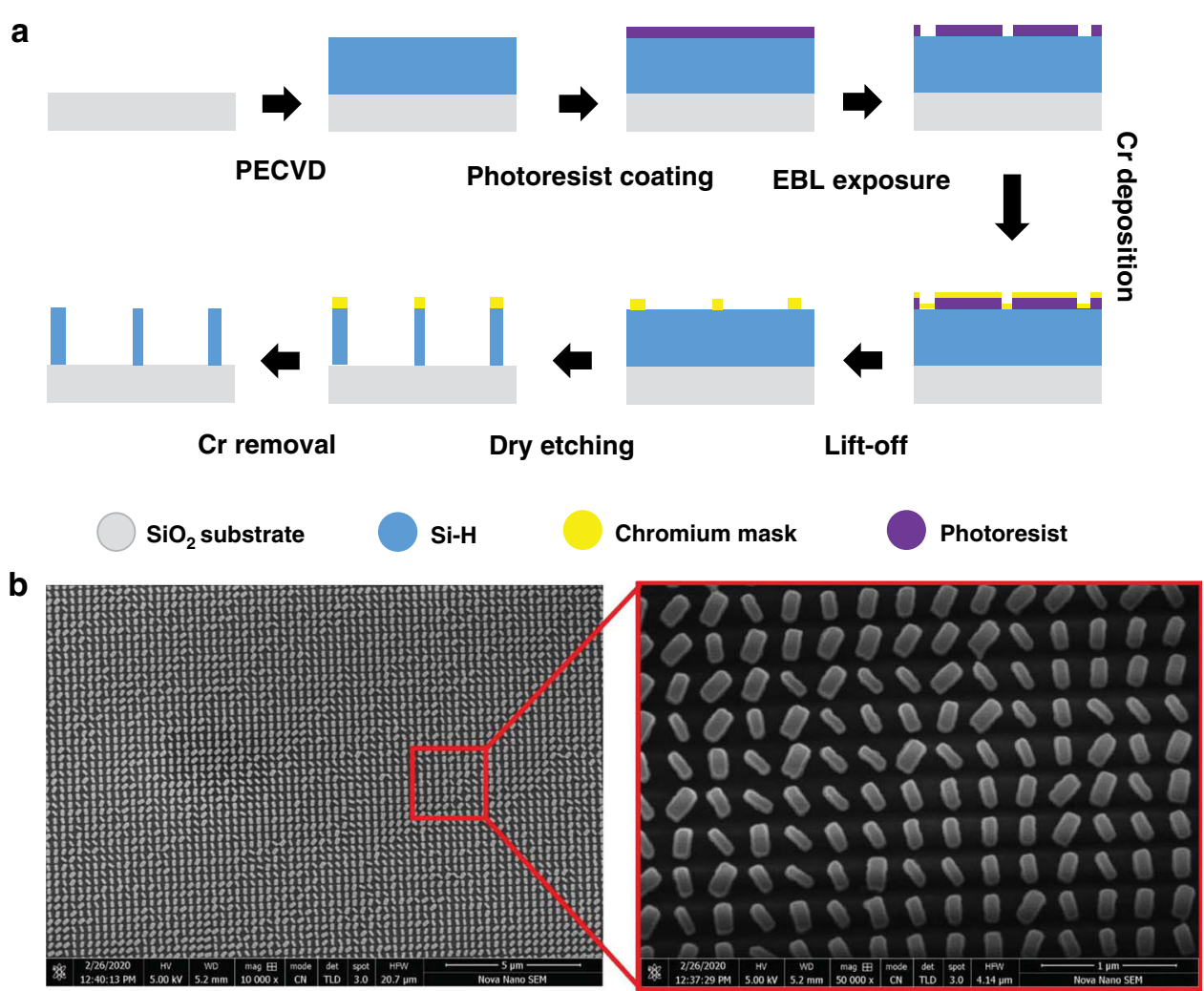

Fig. 5 Fabrication steps and SEM images of the metasurface. a The metahologram was fabricated through the PECVD process. First, an a-Si:H layer was grown on top of a $500-\mu \mathrm{m}$ thick $\mathrm{SiO}_{2}$ substrate. The deposition rate of a-Si:H was controlled by managing the flow rates of saline $\left(\mathrm{SiH} \mathrm{H}_{4}\right)$ and hydrogen $\left(\mathrm{H}_{2}\right)$ gases. The a-Si:H nanorods were defined by electron-beam lithography on a positive-type photoresist. A 30-nm thick chromium ( $\mathrm{Cr}$ ) layer was deposited followed by the lift-off process. The geometrical arrangement was transferred to a-Si:H using the Cr mask and dry etching. $\mathbf{b}$ SEM images of the designed asymmetric wavefront generation metasurface. The right image shows a magnified view of our fabricated hydrogenated noncrystalline silicon nanoresonators on top of a $\mathrm{SiO}_{2}$ substrate.

high fidelity at the incident wavelength of $632.8 \mathrm{~nm}$. One possible reason for the slight blemish on the output in Fig. $6 \mathrm{~b}$ is the imperfect conversion of the unpolarized incident light into circularly polarized light, which could be resolved using near-perfect polarization filters. The metahologram simultaneously provides a single image for forward and backward illumination. The polarization conversion ratio (PCR) is also a basic parameter to define how much incident light is converted into cross-polarized light. The PCR is described as the ratio of the crosspolarized component to the sum of the cross- and copolarized components. The simulated average PCR for the proposed metasurface is $91 \%$, depicted by the blue line in Fig. $6 \mathrm{~d}$, while the measured PCR is $88 \%$, highlighted by the brown dot.

\section{Conclusion}

In summary, a transmission-type multifunctional metasurface, which is CMOS process compatible and operates in the visible domain at a wavelength of $632.8 \mathrm{~nm}$, has been demonstrated. A low-loss material,
a-Si:H, with a high-refractive index and a low extinction coefficient for visible light was used, which relaxes the fabrication challenges faced when using other dielectric materials. Moreover, asymmetric spin-orbit interactions were manipulated such that they enabled asymmetric wavefront generation. The proposed technique controls the wavefront and independent phase modulation simultaneously by exploiting the whole metasurface array, in contrast to conventional symmetric helicity-multiplexed metasurfaces. We designed and fabricated a $300 \times$ $300 \mu \mathrm{m}^{2}$ metasurface to produce two central-symmetric holographic images of the POSTECH and ITU logos at a focal length of $150 \mu \mathrm{m}$. Under red light $(632.8 \mathrm{~nm})$ incidence, the measured transmission efficiency of our proposed metasurface was $59 \%$. Therefore, a high transmission was attained in the visible spectrum using a-Si:H without compromising its performance. Furthermore, advanced metasurface design methodologies, such as wavelength-decoupled metasurface fabrication ${ }^{51}$, phaseintensity complex modulation ${ }^{52}$, chiro-optical metaholograms $^{53}$, and complex-amplitude modulation by orbital 

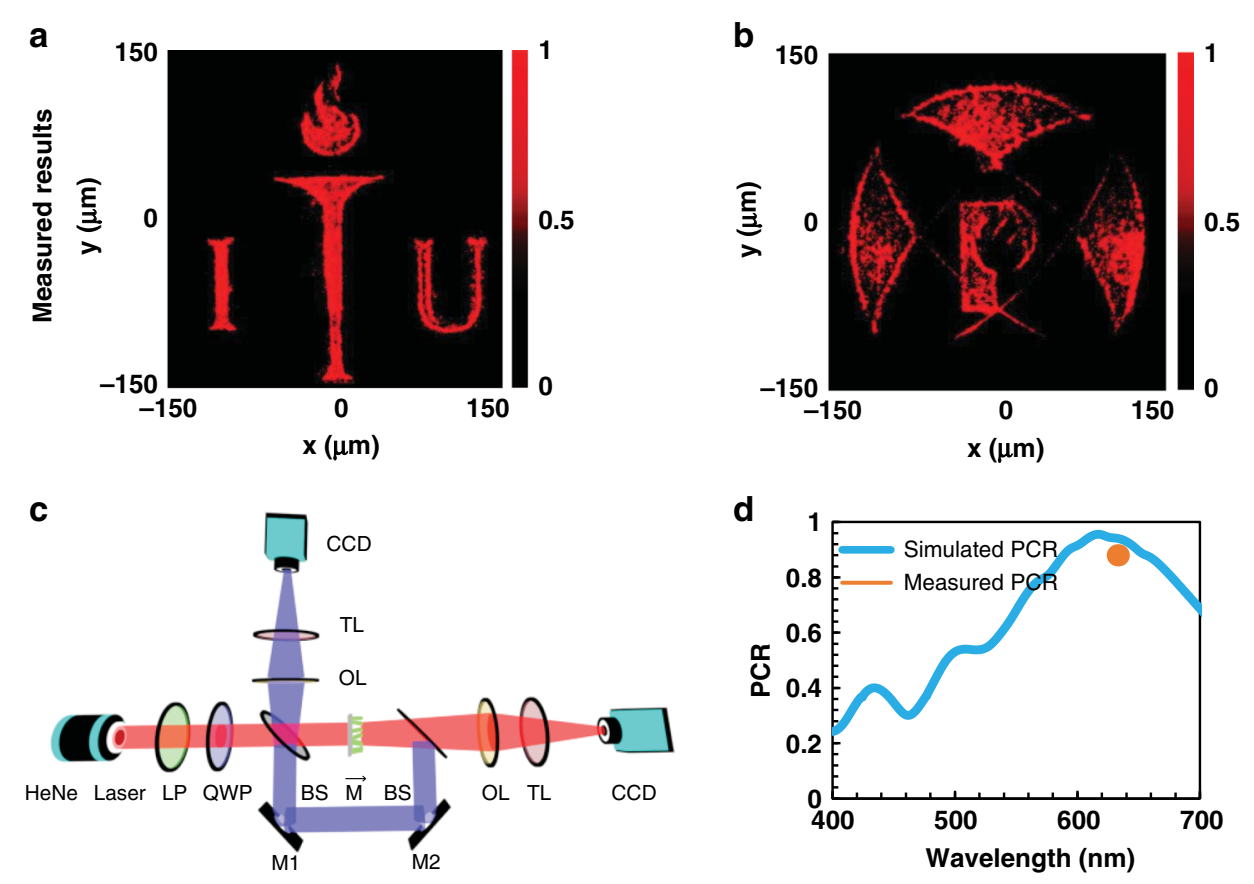

Fig. 6 Optically measured results obtained from the asymmetric wavefront generation metasurface. Optically measured results obtained after characterization of the $300 \times 300 \mu^{2}$ metahologram, displaying the generation of two holographic images for RCP light incident in the a backward and $\mathbf{b}$ forward directions (see supplementary video part ' $b$ '). c Optical characterization setup used in the amplitude profile measurement of the metahologram ${ }^{50}$. d Polarization conversion ratio (PCR) of the proposed metasurface. At the designed wavelength of $632.8 \mathrm{~nm}$, the simulated PCR is $91 \%$.

angular momentum ${ }^{54-56}$, will enable multicolor and high-resolution bifacial metaholograms. Additionally, recently developed nanoimprinting-based scalable manufacturing techniques will further accelerate the mass production of metahologram devices ${ }^{57,58}$. We believe that this is a significant step towards the implementation of multifunctional, CMOS-compatible, and integrated photonic devices such as image sensors ${ }^{59}$. Also, once the metaholograms are further integrated with active platforms such as liquid crystals, real-time dynamic and highresolution metaholographic displays will be realized ${ }^{60}$.

\section{Acknowledgements}

This work was financially supported by the LGD-SNU incubation program funded by LG Display and the National Research Foundation of Korea (NRF) grants (NRF-2019R1A2C3003129, CAMM-2019M3A6B3030637, NRF2019R1 A5A8080290) funded by the Ministry of Science and ICT (MSIT) of the Korean government. M.Q.M acknowledges a research grant by Higher Education Commission (HEC) of Pakistan through National Research Program for Universities (NRPU) [Project No. 10177/Punjab/ NRPU/R\&D/HEC/ 2017] to support this work. M.Q.M., T.T., K.R., U.Y., and M.Z. acknowledge their internal research grants from ITU. M.A.A. acknowledges the Pre-Doctoral Fellowship from ITU. I.K. acknowledges the NRF Sejong Science fellowship (NRF-2021R1C1C2004291) funded by the MSIT of the Korean government.

\section{Author details}

${ }^{1}$ NanoTech Lab, Department of Electrical Engineering, Information Technology University (ITU) of the Punjab, Ferozepur Road, Lahore 54600, Pakistan.

${ }^{2}$ Department of Mechanical Engineering, Pohang University of Science and Technology (POSTECH), Pohang 37673, Republic of Korea. ${ }^{3}$ Department of
Physics, Lahore University of Management Sciences (LUMS), Lahore 54792 , Pakistan. ${ }^{4}$ Department of Chemical Engineering, Pohang University of Science and Technology (POSTECH), Pohang 37673, Republic of Korea. ${ }^{5}$ National Institute of Nanomaterials and Technology (NINT), Pohang 37673, Republic of Korea

\section{Author contributions}

J.R. and M.Q.M. conceived the idea and started the project. M.A.A. and M.A.N. established the process and developed the experiments. I.K., J.K., and D.K.O. fabricated the devices. M.A.A. and I.K. performed the characterizations of the fabricated devices. M.A.A., M.A.N., I.K., T.B., J.R., and M.Q.M. analyzed the data and mainly wrote the manuscript. K.R., T.T., M.Z., U.Y., M.S., and M.S.A. provided theoretical advice. J.R. and M.Q.M. guided the entire research. All authors contributed to the discussion and confirmed the final manuscript.

\section{Conflict of interest}

The authors declare that they have no conflict of interest.

Supplementary information accompanies this paper at https://doi.org/ 10.1038/s41378-020-00226-X.

Received: 26 August 2020 Revised: 24 September 2020 Accepted: 4 October 2020

Published online: 03 March 2021

\section{References}

1. Li, Z. et al. Dielectric meta-holograms enabled with dual magnetic resonances in visible light. ACS Nano 11, 9382-9389 (2017).

2. Ansari, M. A., Tauqeer, T., Zubair, M., \& Mehmood, M. Q. Breaking polarisationbandwidth trade-off in dielectric metasurface for unpolarised white light. 
Nanophotonics 9, 963-971. https://www.degruyter.com/view/journals/ nanoph/9/4/article-p963.xml (2020).

3. Ryan, C. G. et al. A wideband transmitarray using dual-resonant double square rings. IEEE Trans. Antennas Propag. 58, 1486-1493 (2010).

4. Mehmood, M. Q. et al. Visible-frequency metasurface for structuring and spatially multiplexing optical vortices. Adv. Mater. 28, 2533-2539 (2016).

5. Mahmood, N. et al. Polarisation insensitive multifunctional metasurfaces based on all-dielectric nanowaveguides. Nanoscale 10, 18323-18330 (2018).

6. Li, Z. et al. Full-space cloud of random points with a scrambling metasurface. Light Sci. Appl. 7, 63 (2018).

7. Kawano, K. et al. Holographic recording and retrieval of polarized light by use of polyester containing cyanoazobenzene units in the side chain. Opt. Lett. 24 1269-1271 (1999).

8. Slinger, C., Cameron, C. \& Stanley, M. Computer-generated holography as a generic display technology. Computer 38, 46-53 (2005).

9. Wen, D. et al. Helicity multiplexed broadband metasurface holograms. Nat. Commun. 6, 8241 (2015).

10. Zheng, G. et al. Metasurface holograms reaching $80 \%$ efficiency. Nat. Nanotechnol. 10, 308-312 (2015).

11. Ni, X., Kildishev, A. V. \& Shalaev, V. M. Metasurface holograms for visible light. Nat. Commun. 4, 2807 (2013).

12. Huang, $\mathrm{K}$. et al. Ultrahigh-capacity non-periodic photon sieves operating in visible light. Nat. Commun. 6, 7059 (2015).

13. Li, X. et al. Multicolor 3D meta-holography by broadband plasmonic modulation. Sci. Adv. 2, e1601102 (2016).

14. $\mathrm{Pu}, \mathrm{M}$. et al. Catenary optics for achromatic generation of perfect optical angular momentum. Sci. Adv. 1, e1500396 (2015).

15. Lin, D., Fan, P., Hasman, E. \& Brongersma, M. L. Dielectric gradient metasurface optical elements. Science 345, 298-302 (2014).

16. Boltasseva, A. \& Atwater, H. A. Low-loss plasmonic metamaterials. Science $\mathbf{3 3 1}$ 290-291 (2011).

17. Schuller, J. A., Zia, R., Taubner, T. \& Brongersma, M. L. Dielectric metamaterials based on electric and magnetic resonances of silicon carbide particles. Phys. Rev. Lett. 99, 107401 (2007).

18. Genevet, P., Capasso, F., Aieta, F., Khorasaninejad, M. \& Devlin, R. Recent advances in planar optics: from plasmonic to dielectric metasurfaces. Optica 4, 139-152 (2017).

19. Hsiao, H. H., Chu, C. H. \& Tsai, D. P. Fundamentals and applications of metasurfaces. Small Methods 1, 1600064 (2017).

20. Yang, Y., Kravchenko, I. I., Briggs, D. P. \& Valentine, J. All-dielectric metasurface analogue of electromagnetically induced transparency. Nat. Commun. 5, 5734 (2014).

21. Zhou, Z. et al. Efficient silicon metasurfaces for visible light. ACS Photonics 4, 544-551 (2017).

22. Pierce, D. T. \& Spicer, W. E. Electronic structure of amorphous Si from photoemission and optical studies. Phys. Rev. B 5, 3017 (1972).

23. Kawashima, T., Yoshikawa, H., Adachi, S., Fuke, S. \& Ohtsuka, K. Optical properties of hexagonal GaN. J. Appl. Phys. 82, 3528-3535 (1997).

24. Siefke, T. et al. Materials pushing the application limits of wire grid polarizers further into the deep ultraviolet spectral range. Adv. Opt. Mater. 4, 1780-1786 (2016).

25. Chen, B. H. et al. GaN metalens for pixel-level full-color routing at visible light. Nano Lett. 17, 6345-6352 (2017).

26. Devlin, R. C., Khorasaninejad, M., Chen, W. T., Oh, J. \& Capasso, F. Broadband high-efficiency dielectric metasurfaces for the visible spectrum. Proc. Natl Acad. Sci. USA 113, 10473-10478 (2016).

27. Song, Q. et al. Ptychography retrieval of fully polarized holograms from geometric-phase metasurfaces. Nat. Commun. 11, 2651 (2020).

28. Khorasaninejad, M. et al. Metalenses at visible wavelengths: diffraction-limited focusing and subwavelength resolution imaging. Science 352, 1190-1194 (2016).

29. Wang, S. et al. A broadband achromatic metalens in the visible. Nat. Nanotechnol. 13, 227-232 (2018).

30. Ansari, M. A. et al. A spin-encoded all-dielectric metahologram for visible light. Laser Photonics Rev. 13, 1900065 (2019).

31. Ansari, M. A. et al. Engineering spin and antiferromagnetic resonances to realize an efficient direction-multiplexed visible meta-hologram. Nanoscale Horiz. 5, 57-64 (2020).
32. Huang, Y.-W. et al. Gate-tunable conducting oxide metasurfaces. Nano Lett. 16 5319-5325 (2016)

33. Colburn, S. et al. Broadband transparent and CMOS-compatible flat optics with silicon nitride metasurfaces. Opt. Mater. Exp. 8, 2330-2344 (2018).

34. Zhan, A. et al. Low-contrast dielectric metasurface optics. ACS Photonics $\mathbf{3}$, 209-2014 (2016).

35. Yoon, G., Lee, D., Nam, K. T. \& Rho, J. Pragmatic metasurface hologram at visible wavelength: the balance between diffraction efficiency and fabrication compatibility. ACS Photonics 5, 1643-1647 (2017).

36. Liu, J. et al. High-efficiency mutual dual-band asymmetric transmission of circularly polarized waves with few-layer anisotropic metasurfaces. Adv. Opt. Mater. 4, 2028-2034 (2016).

37. Wang, $Y . H$. et al. Experimental verification of asymmetric transmission in continuous omega-shaped metamaterials. RSC Adv. 8, 38556-38561 (2018).

38. Sung, J., Lee, G. Y., Choi, C., Hong, J. \& Lee, B. Single-layer bifacial metasurface: full-space visible light control. Adv. Opt. Mater. 7, 1801748 (2019).

39. Liu, J. et al. High-efficiency mutual dual-band asymmetric transmission of circularly polarized waves with few-layer anisotropic metasurfaces. Adv. Opt Mater. 4, 2028-2034 (2016).

40. Zhang, L. et al. Transmission-reflection-integrated multifunctional coding metasurface for full-space controls of electromagnetic waves. Adv. Funct. Mater. 28, 1802205 (2018).

41. Chen, Y., Gao, J. \& Yang, X. Direction-controlled bifunctional metasurface polarizers. Laser Photonics Rev. 12, 1800198 (2018).

42. Chen, Y., Yang, X. \& Gao, J. 3D Janus plasmonic helical nanoapertures for polarization-encrypted data storage. Light Sci. Appl. 8, 45 (2019).

43. Chen, K. et al. Directional Janus metasurface. Adv. Mater. 32, 1906352 (2020).

44. Zhang, F. et al. All-dielectric metasurfaces for simultaneous giant circular asymmetric transmission and wavefront shaping based on asymmetric photonic spin-orbit interactions. Adv. Funct. Mater. 27, 1704295 (2017).

45. Mahmood, N. et al. Twisted non-diffracting beams through all dielectric metaaxicons. Nanoscale 11, 20571-20578 (2019).

46. Legesse, M., Nolan, M. \& Fagas, G. Revisiting the dependence of the optical and mobility gaps of hydrogenated amorphous silicon on hydrogen concentration. J. Phys. Chem. C. 117, 23956-23963 (2013).

47. Huang, K. et al. Silicon multi-meta-holograms for the broadband visible light. Laser Photonics Rev. 10, 500-509 (2016).

48. Zhang, F., Pu, M., Luo, J., Yu, H. \& Luo, X. Symmetry breaking of photonic spinorbit interactions in metasurfaces. Opto Electron. Eng. 44, 319-325 (2017).

49. Dong, B. Z., Zhang, Y., Gu, B. Y. \& Yang, G. Z. Numerical investigation of phase retrieval in a fractional Fourier transform. J. Opt. Soc. Am. A 14, 2709-2714 (1997).

50. Kim, I., Lee, D. \& Rho, J. Demonstration of spin- and direction-multiplexed alldielectric visible metaholograms. J. Vis. Exp. 163, e61334 (2020).

51. Yoon, G. et al. Wavelength-decoupled geometric metasurfaces by arbitrary dispersion control. Commun. Phys. 2, 129 (2019).

52. Lee, G.-Y. et al. Complete amplitude and phase control of light using broadband holographic metasurfaces. Nanoscale 10, 4237-4245 (2018).

53. Rana, A. S. et al. Planar achiral metasurfaces-induced anomalous chiroptical effect of optical spin isolation. ACS Appl. Mater. Interfaces 12,48899-48909 (2020).

54. Ren, $\mathrm{H}$. et al. Metasurface orbital angular momentum holography. Nat Commun. 10, 2986 (2019).

55. Fang, X., Ren, H. \& Gu, M. Orbital angular momentum holography for highsecurity encryption. Nat. Photonincs 14, 102-108 (2020).

56. Ren, $H$. et al. Complex-amplitude metasurface-based orbital angular momentum holography in momentum space. Nat. Nanotechnol. 15, 948-955 (2020).

57. Kim, K., Yoon, G., Baek, S., Rho, J. \& Lee, H. Facile nanocasting of dielectric metasurfaces with sub-100nm resolution. ACS Appl. Mater. Inter. 11, 26109-26115 (2019).

58. Yoon, G., Kim, K., Huh, D., Lee, H. \& Rho, J. Single-step manufacturing of hierarchical dielectric metalens in the visible. Nat. Commun. 11, 2268 (2020).

59. Jeong, $\mathrm{H}$. et al. Emerging advanced metasurfaces: Alternatives to conventional bulk optical devices. Microelectron. Eng. 220, 111146 (2020).

60. Kim, I. et al. Stimuli-responsive dynamic meta-holographic displays with designer liquid crystal modulators. Adv. Mater. 32, 2004664 (2020). 